\section{A new brain organoid model to study Parkinson's Disease}

\author{
Silvia Bolognin, ${ }^{1}$ Lisa M. Smits, ${ }^{1}$ \\ Sarah L. Nickels, ${ }^{1}$ Stefano Magni, ${ }^{1}$ \\ Paul Antony, ${ }^{1}$ Kamil Grzyb, ${ }^{1}$ \\ Rejko Krüger, ${ }^{1-3}$ Alexander Skupin, ${ }^{1}$ \\ Jens C. Schwamborn1 \\ ${ }^{1}$ Luxembourg Centre for Systems \\ Biomedicine, University of Luxembourg, \\ Belvaux; ${ }^{2}$ Transversal Translational \\ Medicine, Luxembourg Institute of \\ Health, Strassen; ${ }^{3}$ Centre Hospitalier de \\ Luxembourg, Luxembourg City, \\ Luxembourg
}

\begin{abstract}
Human midbrain organoid models represent a new tool to study the underlaying etiology of Parkinson's disease in physiological conditions.
\end{abstract}

\section{Introduction}

Parkinson's Disease (PD) is the second most prevalent neurodegenerative disorder in the aging population. It is characterized by the progressive loss of dopaminergic neurons in the substantia nigra of the patients. The cause of the disease is unknown and consequently, no diseasemodifying drug is currently available. The difficulty in translating basic research into the clinic is partly due to the excessive reliance on animal models, which do not faithfully recapitulate the human pathology. The field of human brain organoids has recently gained increasing attention for the ability to harness the key capability of stem cells to self-organize into organ-like structures. This represents an unparallel opportunity to study neurodegenerative mechanisms in human models. We aimed at optimizing a brain organoid model recapitulating key features of the midbrain region of the human brain. Therefore, we used stem cells from patients carrying the PD-associated familial mutation LRRK2-G2019S to evaluate whether dopaminergic neuron impairment could be identified.

\section{Materials and Methods}

We generated Midbrain Organoids (MOs) from Induced Pluripotent Stem Cells (iPSCs) derived from two PD patients car- rying the LRRK2-G2019S mutation and from two healthy individuals, both with their isogenic controls. ${ }^{1}$ The organoids were cultured for 35 and 70 days before being analyzed via immunofluorescence. Experiments were performed with three independently generated organoid cultures from three different cell lines $(n=9)$. Unpaired $t$ test with Welch's correction or nonparametric Kolmogorov-Smirnov test was performed to evaluate statistical significance. Single cell RNA sequencing (scRNA-seq) was also performed in MOs generated from a healthy individual as well as its isogenic control where the LRRK2G2019S mutation was inserted.2

\section{Results}

MOs showed abundant expression of dopaminergic neurons, which produced and secreted the neurotransmitter dopamine. Accumulation of neuromelanin was also observed. Besides dopaminergic neurons, GABAergic, glutamatergic, and serotoninergic neurons together with astrocytes were identified, confirming that the MO is a complex system, where the cellular diversity of the substantia nigra is well represented. When MOs carrying the LRRK2-G2019S mutation were generated, we observed a statistically significant decrease of dopaminergic neurons after 35 days of cultivation compared to controls. High-content image analysis allowed the identification of neuronal subtypes based on the pattern of different expression markers. The number of astrocytes was also impaired, suggesting a deleterious effect of the LRRK2 mutation on both neurons and astrocytes. Further efforts will be necessary to advance the model by further integrating microglia and vasculature.

\section{Discussion and Conclusions}

These results show that MOs are suitable to study PD as they model the tissue complexity of the human substantia nigra. Our implemented protocol1,3 gives rise to reproducible MOs, which can be used for phenotyping and pharmacological screenings. The use of MOs, carrying mutations linked to familial PD, constitutes a valuable resource to study the functionality of both dopaminergic neurons and astrocytes and their contribution to the disease progression. MOs harboring the LRRK2-G2019S mutations show dopaminergic impairment that can be captured via high content image analysis and scRNA-seq. Our platform
Correspondence: Silvia Bolognin, Luxembourg Centre for Systems Biomedicine, University of Luxembourg, Belvaux.

E-mail: silvia.bolognin@uni.lu

Key words: Parkinson's disease; organoids; LRRK2; neurons.

Acknowledgments: SB is supported by the Fonds National de la Recherche (FNR) (CORE JR C-C19/BM/13626885/IDeM)

Disclosures: JCS and SB are shareholders of the biotech company OrganoTherapeutics SARL.

Conference presentation: This paper was presented at the Third Centro 3R Annual Meeting - L'era delle 3R: modelli in silico, in vitro e in vivo per promuovere la ricerca traslazionale 30 September - 1 October 2021, Evento online organizzato dal Politecnico di Torino.

Received for publication: 9 July 2021

Accepted for publication: 7 September 2021.

This work is licensed under a Creative Commons Attribution NonCommercial 4.0 License (CC BY-NC 4.0).

(C) Copyright: the Author(s), 2021

Licensee PAGEPress, Italy

Biomedical Science and Engineering 2021; 4(s1):137 doi:10.4081/bse.2021.137

allows the identification of tractable PD cellular phenotypes which can be targeted with drug candidates.

\section{References}

1. Smits LM, Reinhardt L, Reinhardt P, et al. Modeling Parkinson's disease in midbrain-like organoids. npj Parkinsons Dis 2019;5:5

2. Smits LM, Magni S, Kinugawa K, et al Single-cell transcriptomics reveals multiple neuronal cell types in human midbrain-specific organoids. Cell Tissue Res 2020;382:463-76.

3. Nickels SL, Modamio J, MendesPinheiro B, et al. Reproducible generation of human midbrain organoids for in vitro modeling of Parkinson's disease. Stem Cell Res 2020;46:101870. 